\title{
Ant Colony Optimization Based on Adaptive Volatility Rate of Pheromone Trail
}

\author{
Zhaoquan CAI ${ }^{1}$, Han HUANG ${ }^{2,3}$, Yong QIN ${ }^{4}$, Xianheng MA $^{2}$ \\ ${ }^{1}$ Educational Technology Center, Huizhou University, Huizhou, China \\ ${ }^{2}$ School of Software Engineering, South China University of Technology, Guangzhou, China \\ ${ }^{3}$ State Key Lab for Novel Software Technology, Nanjing University, Nanjing, China \\ ${ }^{4}$ Center of Information and Network, Maoming University, Maoming, China \\ Email: \{gdzqcai, bssthh\}@163.com \\ Received May 25, 2009; revised July 15, 2009; accepted August 13, 2009
}

\begin{abstract}
Ant colony optimization (ACO) has been proved to be one of the best performing algorithms for NP-hard problems as TSP. The volatility rate of pheromone trail is one of the main parameters in ACO algorithms. It is usually set experimentally in the literatures for the application of ACO. The present paper first proposes an adaptive strategy for the volatility rate of pheromone trail according to the quality of the solutions found by artificial ants. Second, the strategy is combined with the setting of other parameters to form a new ACO method. Then, the proposed algorithm can be proved to converge to the global optimal solution. Finally, the experimental results of computing traveling salesman problems and film-copy deliverer problems also indicate that the proposed ACO approach is more effective than other ant methods and non-ant methods.
\end{abstract}

Keywords: Ant Colony Optimization (ACO), Adaptive Volatility Rate, Pheromone Trail

\section{Introduction}

ACO was first proposed by M. Dorigo and his colleagues as a multi-agent approach to deal with difficult combinatorial optimization problems such as TSP [1]. Since then, a number of applications to the NP-hard problems have shown the effectiveness of ACO [1]. Up till now, Ant Colony System (ACS) [2] and MAX-MIN Ant System (MMAS) [3] are so successful and classical that their strategies such as pheromone global-local update and Maximum-Minimum of pheromone are widely used in recent research [1].

The main parameters of ACO may conclude: $k, \rho$, $\alpha$ and $\beta$, where $k$ is the number of artificial ants used for solution construction, $\rho$ is the parameter for volatility of pheromone trail and $\alpha, \beta$ determines the relative importance of pheromone value and heuristic information $[2,4,5]$. All of the parameters are usually set with experimental methods in the application of ACO [5-7]. For the adaptive parameter setting, M. Dorigo and L.M. Gambardella presented a formula for the optimal number of ants $k$ based on the value of $\rho$ and $q_{0}$ in ant colony system. I. Watanabe and S. Matsui proposed an adaptive control mechanism of the parameter candidate sets based on the pheromone concentrations [8]. M. L., Pilat, and T. White put forward the ACSGA-TSP algorithm [9] with an adaptive evolutionary parameters $\beta, \rho, q_{0}$ and gave the experimental values of these parameters for some TSP problems. For the parameters $\alpha$ and $\beta$, which regulate the relative importance of pheromone trail and closeness [10], H. Huang proposed a dynamic strategy for a bi-directional searching ant colony system [11]. However, other parameters should be set experimentally.

This paper presents a trial work of setting the parameters of ACO adaptively. First, a tuning rule for $\rho$ is designed based on the quality of the solution constructed by artificial ants. Then, we introduce the adaptive $\rho$ to form a new ACO algorithm, which is tested to compute several benchmark instances of traveling sales-man problem and film-copy deliverer problem. Finally, the experimental result indicates that the new ACS with adaptive $\rho$ performs better than GA [12], ACO [13] and ACS [2,14]. Furthermore, the convergence of the proposed ACO algorithm is proved. 


\section{Adaptive Volatility Rate of Pheromone Trail}

The framework of ACO [1-2] is inspired by the ants' foraging behavior in selecting the shortest path between the nest and the food. Each ant builds a tour (i.e. a feasible solution to the TSP) by repeatedly applying a stochastic greedy rule (the state transition rule) as Equation (1) shows.

$$
P_{g s}^{m}(t)= \begin{cases}\frac{\left[\tau_{g s}(t)\right]^{\alpha}\left[\eta_{g s}\right]^{\beta(g, t)}}{\sum_{r \in J_{m}(g)}\left[\tau_{g r}(t)\right]^{\alpha}\left[\eta_{g r}\right]^{\beta(g, t)}} & \text { if } \quad s \in J_{k}(g) \\ 0 & \text { otherwise }\end{cases}
$$

where $P_{g s}^{m}$ is the probability with which the ant $m$ chooses to move from city $g$ to city $s$ in iteration $t$, $\tau$ is the pheromone, $\eta=1 / d$ is the reciprocal of distance $d_{g s}$, and $J_{m}(g)$ is the set of cities not having been visited yet when ant $m$ is at city $g$.

After constructing its tour, an artificial ant also modifies the amount of pheromone on the visited edges by applying the pheromone updating rule. The rule is designed so that it tends to give more pheromone to the edges which should be visited by ants. The classical pheromone updating rule is:

$$
\tau_{g s}(t+1)=(1-\rho) \tau_{g s}(t)+\rho \Delta \tau_{g s}(t)
$$

where $\Delta \tau_{g s}(t)$ is the increment for the pheromone of edge $(g, s)$ at the $t$-th iteration, and $\rho$ is the volatility rate of the pheromone trail. The optimal $\rho$ was set $\rho=0.1$ experimentally $[1,2,4]$, which means that 90 per cent of the original pheromone trail remains and its 10 per cent is replaced by the increment.

In order to update the pheromone according to the quality of solutions found by ants, an adaptive rule for volatility of the pheromone trail is designed as follows:

$$
\rho_{m}=L_{m}^{-1} /\left(L_{m}^{-1}+L_{P}^{-1}\right)
$$

where $L_{m}$ is the length of the solution $S_{m}$ found by ant $m$, and $L_{P}$ is the length of the solution $S_{P}$ built based on the pheromone matrix, shown as Equation (4).

$$
s=\arg \max _{u \in J_{m}(r)}\{[\tau(r, u)\}
$$

where $s$ is the city selected as the next one to city $r$ for any $(r, s) \in S_{P}$.

The motivation of the proposed rule is: better solutions should contribute more pheromone, and the worse ones contribute less. We will use this rule to design a new $\mathrm{ACO}$ algorithm in the following section.

\section{An ACO Algorithm with the Adaptive Parameter}

In this section, a new ACO algorithm with the adaptive rule (shown as Equation 3) is introduced as follows:

Algorithm new ACO

input: An instance of TSP or FDP problems

Initialize solutions and pheromone value.

$S_{\text {best }} \leftarrow N U L L$.

while termination conditions not met do

Construct $S_{P}$ tificial ants\}

for $i=1$ to $k$ do $\{k$ is the number of ar-

$S_{i} \leftarrow$ ConstructSolution $(t)$.

$\rho_{i}$ is calculated based on $S_{i}$.

if $\quad\left(\operatorname{Length}\left(S_{i}\right)<\operatorname{Length}\left(S_{b e s t}\right) \quad\right.$ or

$\left(S_{\text {best }}=N U L L\right)$ then

$$
S_{\text {best }} \leftarrow S_{i}
$$

\section{Endif}

Endfor

$\rho_{\text {best }}$ is calculated based on $S_{\text {best }}$.

Carry out the pheromone updating rule with $\rho_{i}(i=1, \ldots, k)$ and $\rho_{\text {best }}$.

\section{Endwhile}

Output: $S_{\text {best }}$.

\section{End_Algorithm}

The framework of the proposed algorithm is similar to ant colony system (ACS) [2], so are the initialization, solution construction and setting of the parameters $q_{0}=0.9, k=10, \alpha=1$ and $\beta=2$. There is only an updating rule in the algorithm shown as Equation 5 and 6.

$$
\tau_{g s}(t+1)=\left(1-\rho_{i}\right) \tau_{g s}(t)+\rho_{i} L_{i}^{-1}
$$

where $\forall(g, s) \in S_{i}$ and $\rho_{i}=L_{i}^{-1} /\left(L_{i}^{-1}+L_{P}^{-1}\right)$ for the $t$-th iteration.

$$
\tau_{g s}(t+1)=\left(1-\rho_{\text {best }}\right) \tau_{g s}(t)+\rho_{\text {best }} L_{\text {best }}{ }^{-1}
$$

where $\forall(g, s) \in S_{\text {best }}$ and $\rho_{\text {best }}=L_{\text {best }}^{-1} /\left(L_{\text {best }}^{-1}+L_{P}^{-1}\right)$ for the $t$-th iteration.

\section{Convergence of the Proposed Algorithm}

In this section, we give the convergence proof of the new ACO algorithm.

Given an arbitrary path $(g, s)$,

$\tau_{g s}(t) \leq\left(1-\rho_{1}\right) \tau_{g r}(t)+\rho_{1} U \leq\left(1-\rho_{1}\right)^{t^{\prime}} \tau_{g s}(0)+\frac{1-\left(1-\rho_{1}\right)^{t^{\prime}}}{1-\left(1-\rho_{1}\right)} \rho_{1} U$ 
where $0<t^{\prime} \leq t, \quad \rho_{1}=L_{\text {min }}^{-1} /\left(L_{\min }^{-1}+L_{\max }^{-1}\right), \quad U=\max$ $\left\{\tau_{g s}(0),\left(L_{\min }\right)^{-1}\right\}, L_{\max }$ is the length of the worst tour and $L_{\min }$ is the length of optimal tours.

$\tau_{g s}(t) \geq\left(1-\rho_{2}\right) \tau_{g r}(t)+\rho_{2} D \leq\left(1-\rho_{2}\right)^{t^{\prime}} \tau_{g s}(0)+\frac{1-\left(1-\rho_{2}\right)^{t^{\prime}}}{1-\left(1-\rho_{2}\right)} \rho_{2} D$

where $0<t^{\prime} \leq t, \quad \rho_{2}=L_{\max }^{-1} /\left(L_{\text {max }}^{-1}+L_{\min }^{-1}\right), \quad D=\min$ $\left\{\tau_{g s}(0),\left(L_{\max }\right)^{-1}\right\}$.

Because $0<\rho_{1}, \rho_{2}<1, D \leq \tau_{g s}(t) \leq U$ when $t \rightarrow \infty$.

Therefore, $\tau_{g s}(t)$ has an upper boundary and a lower boundary, we assume $0<P_{\text {low }} \leq \tau_{g s}(t) \leq P_{\text {high }}<+\infty$ without a loss of generality.

When $S^{*}$ is the optimal solution to a $n$-city TSP and $(a, b) \in S^{*}$ as an arbitrary path, the probability $P_{a b}\left(t_{0}\right)$, with which $(a, b)$ is found by artificial ant in iteration $t_{0}\left(t_{0}>0\right)$, can meet:

$$
P_{a b}\left(t_{0}\right) \geq P\left\{q>q_{0}\right\} \frac{\tau_{a b}^{\alpha}\left(t_{0}\right) \cdot \eta_{a b}^{\beta}}{\sum_{j \in J(a)} \tau_{a j}^{\alpha}\left(t_{0}\right) \cdot \eta_{a j}^{\beta}}
$$

where $\quad p_{0}=P\left\{q>q_{0}\right\} \quad[2], \quad \eta_{\min }=\min _{(i, j) \in S^{*}}\left\{\eta_{i j}\right\} \quad$ and $\eta_{\max }=\max _{(i, j) \in S^{*}}\left\{\eta_{i j}\right\}$.

Given $a_{0}=p_{0} \frac{P_{l o w} \cdot \eta_{\min }^{\beta}}{k P_{h i g h} \eta_{\max }^{\beta}}<1$, the probability, by which $S^{*}$ can be found by ants in iteration $t_{0}$, is $P_{S^{*}}\left(t_{0}\right)=\prod_{(a, b) \in S^{*}} P_{a b}\left(n_{0}\right) \geq a_{0}{ }^{n-1}$, where $n$ is the number of cities. The probability, by which $S^{*}$ can never be found from iteration $t_{0}$, is:

$$
\begin{aligned}
& \tilde{P}_{S^{*}}\left(t_{0}\right)=\prod_{t=t_{0}}^{\infty}\left[1-\prod_{(a, b) \in S^{*}} P_{a b}\left(t_{0}\right)\right]^{k} \\
& \leq \prod_{t=t_{0}}^{\infty}\left[1-a_{0}^{n-1}\right]^{k}=0
\end{aligned}
$$

where $k$ is the number of artificial ants and $t_{0}$ can be arbitrary.

Hence, $S^{*}$ can be found by probability one when the iteration $t \rightarrow \infty$, which theoretically confirms the capacity of global optimization of the proposed ACO algorithm.

\section{Numerical Results}

This section indicates the numerical results in the experiment that the proposed ACO algorithm is used to solve TSP problems [15] and FDP problems [14]. Other approaches for the problems ACS [2], ACO [13], GAFDP [12] and ACS-FDP [14] are also tested in the same machines as the comparison with the proposed ACO.

Several TSP instances are computed by ACS [2], ACO [13] and the proposed ACO on a PC with an Intel Pentium 550MBHz Processor and 256MB SDR Memory, and the results are shown in Table 1 . It should be noted that every instance is computed 20 times. The algorithms are both programmed in Visual $\mathrm{C}++6.0$ for Windows System. They would not stop until a better solution could be found in 500 iterations, which is considered as a virtual convergence of the algorithms.

Table 1 shows that the proposed ACO algorithm (PACO) performs better than ACS [2] and ACO [13]. The shortest lengths and the average lengths obtained by PACO are shorter than those found by ACS and ACO in all of the TSP instances. Furthermore, it can be concluded that the standard deviations of the tour lengths obtained by PACO are smaller than those of another algorithms. Therefore, we can conclude that PACO is proved to be more effective and steady than ACS [2] and ACO [13]. Computation time cost of PACO is not less than ACS and ACO in all of the instances because it needs to compute the value of volatility rate $k+1$ times per iteration. Although all optimal tours of TSP problems cannot be found by the tested algorithms, all of the errors for PACO are much less than that for another two ACO approaches. The algorithms may make improvement in solving TSP when reinforcing heuristic strategies like local search like ACS-3opt [2] and MMAS+rs [3] are used.

FDP problem is an extended style of TSP problem. Two FDP instances in the literature [14] are computed by GA-FDP [12], ACS-FDP [14] and the proposed ACOFDP on a PC with an Intel Pentium $400 \mathrm{MBHz}$ Processor and $128 \mathrm{MB}$ EMS memory, and the results are shown in Table 2. It should be noted that every instance is computed 20 times. The algorithms are both programmed in Visual $\mathrm{C}++6.0$ for Windows System. They would not stop until a better solution could be found in 500 iterations, which is considered as a virtual convergence of the algorithms. 
Table 1. Comparison of the results obtained by ACS [2], ACO [3] and the proposed ACO (PACO) in TSP instances.

\begin{tabular}{|c|c|c|c|c|c|}
\hline Problem & Algorithm & best & ave & time(s) & standard deviation \\
\hline \multirow{3}{*}{ kroA100 } & ACS & 21958 & 22088.8 & 65 & 1142.77 \\
\hline & $\mathrm{ACO}$ & 21863 & 22082.5 & 94.6 & 1265.30 \\
\hline & PACO & 21682 & 22076.2 & 117.2 & 549.85 \\
\hline \multirow{3}{*}{ ts 225} & ACS & 130577 & 133195 & 430.6 & 7038.30 \\
\hline & $\mathrm{ACO}$ & 130568 & 132984 & 439.3 & 7652.80 \\
\hline & PACO & 130507 & 131560 & 419.4 & 1434.98 \\
\hline \multirow{3}{*}{ pr226 } & ACS & 84534 & 86913.8 & 378.4 & 4065.25 \\
\hline & $\mathrm{ACO}$ & 83659 & 87215.6 & 523.8 & 5206.70 \\
\hline & PACO & 81967 & 83462.2 & 762.2 & 3103.41 \\
\hline \multirow{3}{*}{$\operatorname{lin} 105$} & ACS & 14883 & 15125.4 & 88.8 & 475.37 \\
\hline & $\mathrm{ACO}$ & 14795 & 15038.4 & 106.6 & 526.43 \\
\hline & PACO & 14736 & 14888 & 112.2 & 211.34 \\
\hline \multirow{3}{*}{ kroB100 } & ACS & 23014 & 23353.8 & 56.2 & 685.79 \\
\hline & $\mathrm{ACO}$ & 22691 & 23468.1 & 102.9 & 702.46 \\
\hline & PACO & 22289 & 22728 & 169.6 & 668.26 \\
\hline \multirow{3}{*}{ kroC100 } & ACS & 21594 & 21942.6 & 54.8 & 509.77 \\
\hline & $\mathrm{ACO}$ & 21236 & 21909.8 & 78.1 & 814.53 \\
\hline & PACO & 20775 & 21598.4 & 114.8 & 414.62 \\
\hline \multirow{3}{*}{$\operatorname{lin} 318$} & ACS & 48554 & 49224.4 & 849.2 & 1785.21 \\
\hline & $\mathrm{ACO}$ & 48282 & 49196.7 & 902.7 & 2459.16 \\
\hline & PACO & 47885 & 49172.8 & 866.8 & 1108.34 \\
\hline
\end{tabular}

Table 2. Comparison of the results obtained by GA-FDP [12], ACS-FDP [14] and the proposed ACO-FDP in FDP instances [14].

\begin{tabular}{ccccc}
\hline \multirow{2}{*}{ Problem } & Algorithm & best & ave & time(s) \\
\hline \multirow{3}{*}{ Problem I } & GA-FDP & 4240.67 & 4261.4 & 153 \\
& ACS-FDP & 4122.33 & 4138.5 & 78 \\
& ACO-FDP & 4122.33 & 4126.2 & 80 \\
\hline \multirow{3}{*}{ Problem II } & GA-FDP & 4208 & 4250.6 & 184 \\
& ACS-FDP & 4163 & 4289.2 & 130 \\
& ACO-FDP & 4163 & 4165.8 & 135 \\
\hline
\end{tabular}

The results in Table 2 indicate that PACO-FDP performs better than GA-FDP [12] and ACS-FDP [14] in the item of average length though it cannot find better solution than ACS-FDP [14]. PACO-FDP can be also considered as the improvement of ACS-FDP because the special strategies [14] are also used in PACO-FDP.

\section{Discussions and Conclusions}

This paper proposed an adaptive rule for volatility rate of pheromone trail, attempting to adjust the pheromone based on the solutions obtained by artificial ants. Thus, a new ACO algorithm is designed with this tuning rule. There is a special pheromone updating rule in the proposed algorithm whose framework is similar to Ant Colony System. Then, the convergence of the proposed ACO algorithm is proved to ensure its capacity of global capacity. Moreover, there are some experimental com- parisons among the proposed ACO approach and other methods [2,12-14] in solving TSP and FDP problems. The results also show the effectiveness of the proposed algorithm.

Further study is suggested to explore the better management for the optimal setting of the parameters of ACO algorithms, which will be very helpful in the application.

\section{Acknowledgements}

This work has been supported by Natural Science Foundation of Guangdong Province (9151600301000001), Key Sci-tech Research Projects of Guangdong Province (2009B010800026), funded project of State Key Lab. for Novel Software Technology of Najing University and student research project (SRP) of South China University of Technology. 


\section{References}

[1] M. Dorigo, G. D. Caro, and L. M. Gambardella, "Ant algorithms for discrete optimization," Massachusetts Institute of Technology, Artificial Life 5, pp. 137-172, 1999.

[2] M. Dorigo and L. M. Gambardella, "Ant colony system: A cooperative learning approach to the traveling salesman problem," IEEE Transactions on Evolutionary Computation, Vol. 1, No. 1, pp. 53-66, 1997.

[3] T. Stützle and H. H. Hoos, "MAX-MIN ant system, future gener," Computer System, Vol. 16, No. 8, pp. 889914, 2000.

[4] M. Dorigo and C. Blum, "Ant colony optimization theory: A survey," Theoretical Computer Science, Vol. 344, pp. 243-278, 2005

[5] A. C. Zecchin, A. R. Simpson, H. R. Maier, and J. B. Nixon, "Parametric study for an ant algorithm applied to water distribution system optimization," IEEE Transactions on Evolutionary Computation, Vol. 9, No. 2, April 2005.

[6] M. Dorigo and L. M. Gambardella, "Ant colonies for the traveling salesman problem," Bio-systems, Vol. 43, pp. 73-81, 1997.

[7] K. M. Sim and W. H. Sun, "Ant colony optimization for routing and load-balancing: Survey and new directions," IEEE Transactions on Systems, Man, and CyberneticsPart A: Systems and Humans, Vol. 33, No. 5, September 2003.

[8] I. Watanabe and S. L. Matsui, "Improving the performance of ACO algorithms by adaptive control of candidate set, evolutionary computation," CEC'03, Vol. 2, pp. 1355-1362, 2003.

[9] M. L. Pilat and T. White, "Using genetic algorithms to optimize ACS-TSP," M. Dorigo et al. (Eds.):ANTS'02, LNCS 2463, pp. 282-287, 2002.

[10] L. M. Gambardella and M. Dorigo, "Ant-Q: A reinforcement learning approach to the traveling salesman problem," Appeared in: Proceedings of ML-95, Twelfth Intern. Conference on Machine Learning, Morgan Kaufmann, pp. 252-260, 1995.

[11] H. Huang and Z. F. Hao, "A bi-directional searching ant colony system," Proceedings of 2006 International Conference on Intelligent Systems and Knowledge Engineering (ISKE'06), April 6-7, 2006.

[12] R. W.Cheng and M. Gen, "Film-copy deliverer problem using genetic algorithms," Computers \& Industrial Engineering, Vo1. 29, No. 1-4, pp. 549-553, 1995

[13] J. Sun, S. W. Xiong, and F. M. Guo, "A new pheromone updating strategy in ant colony optimization," Proceedings of 2004 International Conference on Machine Learning and Cybernetics, Vol. 1, pp. 620-625, 2004.

[14] Z. F.Hao, H. Huang, X. W. Yang, Y. C. Liang, "Solve the film-copy deliverer problem using ant colony system," Proceedings of the Third International Conference on Machine Learning and Cybernetics, Shanghai, 26-29 August 2004.

[15] G. Reinelt, "A traveling salesman problem library," ORSA Journal on Computing, TSPLIB, Vol. 3, No. 4, pp 376-384, 1991. 